ISSN: 1399-0047

journals.iucr.org/d

\title{
Crystal structure and kinetic studies of a tetrameric type II $\beta$-carbonic anhydrase from the pathogenic bacterium Vibrio cholerae
}

\section{Marta Ferraroni, Sonia Del Prete, Daniela Vullo, Clemente Capasso and Claudiu T. Supuran}

Acta Cryst. (2015). D71, 2449-2456

\begin{tabular}{|l|}
\hline IUCr Journals \\
CRYSTALLOGRAPHY JOURNALS ONLINE \\
Copyright C International Union of Crystallography \\
Author(s) of this paper may load this reprint on their own web site or institutional repository provided that \\
this cover page is retained. Republication of this article or its storage in electronic databases other than as \\
specified above is not permitted without prior permission in writing from the IUCr. \\
For further information see http://journals.iucr.org/services/authorrights.html
\end{tabular}


BIOLOGICAL CRYSTALLOGRAPHY

ISSN 1399-0047

Received 9 September 2015

Accepted 5 October 2015

Edited by R. McKenna, University of Florida, USA

Keywords: carbonic anhydrase; $\beta$-carbonic anhydrase; crystal structure; type II CA; Vibrio cholerae.

PDB reference: $\beta$-carbonic anhydrase, 5 cxk

Supporting information: this article has supporting information at journals.iucr.org/d

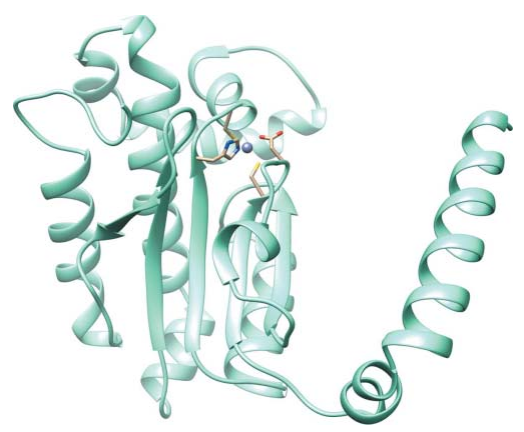

C 2015 International Union of Crystallography

\section{Crystal structure and kinetic studies of a tetrameric type II $\beta$-carbonic anhydrase from the pathogenic bacterium Vibrio cholerae}

\author{
Marta Ferraroni, ${ }^{a *}$ Sonia Del Prete, ${ }^{b}$ Daniela Vullo, ${ }^{a}$ Clemente Capasso ${ }^{b}$ and \\ Claudiu T. Supuran ${ }^{\mathrm{a}, \mathrm{c}}$
}

\begin{abstract}
a'Dipartimento di Chimica 'Ugo Shiff', Polo Scientifico, Università degli Studi di Firenze, Via della Lastruccia 3, Sesto Fiorentino, Firenze, 50019, Italy, ${ }^{\mathbf{b}}$ Istituto di Bioscienze e Biorisorse, CNR, Via Pietro Castellino 111, Napoli, Italy, and ${ }^{\mathbf{c}}$ Sezione di Scienze Farmaceutiche, NEUROFARBA Department, Università degli Studi di Firenze, Via Ugo Schiff 6, Sesto Fiorentino, Firenze, 50019, Italy. ${ }^{*}$ Correspondence e-mail: marta.ferraroni@unifi.it
\end{abstract}

Carbonic anhydrase (CA) is a zinc enzyme that catalyzes the reversible conversion of carbon dioxide to bicarbonate (hydrogen carbonate) and a proton. CAs have been extensively investigated owing to their involvement in numerous physiological and pathological processes. Currently, CA inhibitors are widely used as antiglaucoma, anticancer and anti-obesity drugs and for the treatment of neurological disorders. Recently, the potential use of CA inhibitors to fight infections caused by protozoa, fungi and bacteria has emerged as a new research direction. In this article, the cloning and kinetic characterization of the $\beta$-CA from Vibrio cholerae ( $\mathrm{VchCA} \beta$ ) are reported. The $\mathrm{X}$-ray crystal structure of this new enzyme was solved at $1.9 \AA$ resolution from a crystal that was perfectly merohedrally twinned, revealing a tetrameric type II $\beta$-CA with a closed active site in which the zinc is tetrahedrally coordinated to Cys42, Asp44, His 98 and Cys101. The substrate bicarbonate was found bound in a noncatalytic binding pocket close to the zinc ion, as reported for a few other $\beta$-CAs, such as those from Escherichia coli and Haemophilus influenzae. At pH 8.3, the enzyme showed a significant catalytic activity for the physiological reaction of the hydration of $\mathrm{CO}_{2}$ to bicarbonate and protons, with the following kinetic parameters: a $k_{\text {cat }}$ of $3.34 \times 10^{5} \mathrm{~s}^{-1}$ and a $k_{\text {cat }} / K_{\mathrm{m}}$ of $4.1 \times 10^{7} \mathrm{M}^{-1} \mathrm{~s}^{-1}$. The new enzyme, on the other hand, was poorly inhibited by acetazolamide $\left(K_{\mathrm{i}}\right.$ of $4.5 \mu M)$. As this bacterial pathogen encodes at least three CAs, an $\alpha$-CA, a $\beta$-CA and a $\gamma$-CA, these enzymes probably play an important role in the life cycle and pathogenicity of Vibrio, and it cannot be excluded that interference with their activity may be exploited therapeutically to obtain antibiotics with a different mechanism of action.

\section{Introduction}

Vibrio cholerae is a Gram-negative bacterium found in coastal waters and estuaries that causes cholera, an acute, secretory diarrhoea. Cholera is endemic in over 50 countries and also causes large epidemics, which have recently been increasing in intensity, duration and frequency. Significant antibiotic resistance to $V$. cholerae infection has also been reported (Harris et al., 2012).

Carbonic anhydrases (carbonate hydrolyases; CAs; EC 4.2.1.1) are metalloenzymes that catalyze the interconversion of $\mathrm{CO}_{2}$ and bicarbonate (for reviews, see Aggarwal, Boone et al., 2013; Aggarwal, Kondeti et al., 2013; Supuran, 2012; Capasso \& Supuran, 2015). These enzymes are involved in many physiological processes in microorganisms, such as photosynthesis, respiration and $\mathrm{CO}_{2}$ transport, as well as the metabolism of xenobiotics. Recently, CAs have been cloned and characterized in many pathogenic and nonpathogenic 
bacteria in the search for antibiotics with a novel mechanism of action. It has been demonstrated that CAs are essential for the life cycle of the microorganism and that the conversion of $\mathrm{CO}_{2}$ to bicarbonate plays a role in virulence-gene induction (Capasso \& Supuran, 2013). Bicarbonate in fact induces the expression of the major $V$. cholerae virulence factors by enhancing the activity of ToxT, a regulatory protein that directly activates the transcription of the genes encoding the cholera toxin. This is the first example of a positive effector of ToxT activity, and bicarbonate is likely to be an important in vivo signal that induces $V$. cholerae virulence-gene expression during infection (Abuaita \& Withey, 2009). V. cholerae utilizes the CA system to accumulate bicarbonate in its cells, suggesting a pivotal role of these metalloenzymes in microbial virulence.

Three putative carbonic anhydrases are encoded by the $V$. cholerae bacterium. They belong to three distinct classes: an $\alpha$-CA, a $\beta$-CA and a $\gamma$-CA (Heidelberg et al., 2000). Generally, bacterial genomes only encode CAs belonging to the $\alpha$-CAs, $\beta$-CAS and $\gamma$-CAs. A total of six genetic families of CAs have been discovered to date: $\alpha$-CAs, $\beta$-CAs, $\gamma$-CAs, $\delta$-CAs, $\zeta$-CAs and $\eta$-CAs. Most of them are zinc enzymes, although the $\gamma$ and $\zeta$ classes can have iron(II) or cadmium(II), respectively, in the active site (Lehneck \& Pöggeler, 2014).

The $\beta$-CA class is broadly distributed in nature, including bacteria, algae, plant chloroplasts and invertebrates (Rowlett, 2010). To date, the crystallographic structures of $\beta$-CAs from Pisum sativum (Kimber \& Pai, 2000), the red alga Porphyridium purpureum (Mitsuhashi et al., 2000), Methanobacterium thermoautotrophicum (Strop et al., 2001), Escherichia coli (Cronk et al., 2001), Mycobacterium tuberculosis Rv1284 and Rv3588c (Covarrubias et al., 2005), Haemophilus influenzae (Cronk et al., 2006), Halothiobacillus neapolitanus (Sawaya et al., 2006), the green alga Coccomyxa (Huang et al., 2011), Sordaria macrospora (Lehneck et al., 2014) and Pseudomonas aeruginosa (Pinard et al., 2015) have been published. The biological unit of the structurally characterized $\beta$-CAs appears to be a dimer, a tetramer or an octamer, although the prevalent arrangement seems to be a tetramer or pseudotetramer composed of two pseudo-dimers ( $P$. purpureum and $H$. neapolitanus $\beta$-CAs).

Most of the kinetic properties observed for the $\beta$-CAs are consistent with a catalytic mechanism similar to the well established ping-pong mechanism utilized by the $\alpha$-CAs, in which the catalytically active entity is a zinc-bound water molecule, which ionizes to a hydroxide ion. The first stage of the proposed mechanism involves nucleophilic attack on $\mathrm{CO}_{2}$ by the zinc-bound hydroxide and the exchange of water for bicarbonate at the zinc ion. The second stage is proton transfer from the zinc-bound water to the bulk buffer to regenerate the zinc-bound hydroxide ion. Most of the 15 human CA isoforms known to date have a His residue at position 64 which is actively involved in the proton shuttle and is responsible for converting the zinc-bound water molecule to hydroxide ion. In $\beta$-CAs one or more intramolecular acceptors could play the same role. For example, it has been demonstrated that in $\beta$-carbonic anhydrase from Arabidopsis thaliana residues
His216 and Tyr212 are both important for efficient proton transfer. Tyr212 (Tyr83 in $\beta$-CA from $V$. cholerae) is strictly conserved in the $\beta$-CAs from the Vibrio genus and in other bacterial enzymes (Rowlett et al., 2002).

$\mathrm{X}$-ray crystallographic analysis of $\beta$-CAs revealed two distinct subtypes of this enzyme class based on active-site organization, denoted type I and type II $\beta$-CAs. In the enzymes from $P$. sativum, $M$. thermoautotrophicum and M. tuberculosis Rv1284, the active-site zinc ion is coordinated by one histidine and two cysteine residues, with a fourth coordination site occupied by water or a substrate analogue (the so-called open conformation). In contrast, the other subclass of $\beta$-CAs, exemplified by the structures of the enzymes from $H$. influenzae, E. coli, $P$. purpureum and M. tuberculosis $\mathrm{Rv} 3588 \mathrm{c}$, has a unique zinc-coordination geometry in which the water molecule has been replaced by an aspartate side chain, forming a noncanonical CA active site (the closed conformation). The latter group of CAs (type II $\beta$-CAs) are characterized by little or no $\mathrm{CO}_{2}$ hydration activity at $\mathrm{pH}$ values less than 8.0, compared with the former group (type I $\beta$-CAs) that shows catalytic activity at $\mathrm{pH}$ values from as low as 6.5 to greater than 9.0. Type II $\beta$-CAs also show highly cooperative $\mathrm{pH}$-rate profiles and cooperative inhibition by $\mathrm{HCO}_{3}^{-}$, whereas type I $\beta$-CAs are not inhibited by $\mathrm{HCO}_{3}^{-}$ (Rowlett, 2010).

Therefore, it has been hypothesized that the closed conformation observed in the structures of type II $\beta$-CAs is an allosteric form of the enzyme, the so-called $\mathrm{T}$ state, that is an inactive form present at $\mathrm{pH}$ values below 8.0. This conformation is stabilized by the presence of $\mathrm{HCO}_{3}^{-}$, which was found to bind in a noncatalytic site in E. coli and H. influenzae $\beta$-CAs (Cronk et al., 20068). However, at $\mathrm{pH}$ values of $>8.3$ the closed active site is converted to an open one, with an incoming water molecule replacing the carboxylate moiety of the Asp residue and thus generating the nucleophile used in the catalytic cycle. This has been demonstrated by X-ray crystallography (and kinetic studies) in an elegant work by Jones and coworkers (Covarrubias et al., 2005). Indeed, at this $\mathrm{pH}$ value the carboxylate of the Asp makes a strong interaction with the guanidine/guanidinium moiety of a conserved Arg residue present in all $\beta$-CAs so far investigated (Covarrubias et al., 2005).

Here, we report the kinetic characterization and the crystal structure of the recombinant type II $\beta$-CA from $V$. cholerae determined at a resolution of $1.9 \AA$ with a closed active-site configuration.

\section{Materials and methods}

\subsection{Gene identification}

The identification of the gene encoding $V$. cholerae $\beta$-CA $(\mathrm{V} c h \mathrm{CA} \beta)$ was performed using the NCBI Genome resource (http://www.ncbi.nlm.nih.gov/genome/) selecting the genome of $V$. cholerae. The $\beta$-CA gene of $V$. cholerae was identified by running $B L A S T$ using the $\beta$-CA nucleotide sequence from Brucella suis as the query sequence. The BLAST output file 
Table 1

Summary of data-collection and atomic model-refinement statistics.

Values in parentheses are for the highest resolution shell.

\begin{tabular}{ll}
\hline Data collection & \\
PDB code & $5 \mathrm{cxk}$ \\
Space group & $P 4_{1}$ \\
Unit-cell parameters $(\AA)$ & $a=84.09, c=316.38$ \\
Wavelength $(\AA)$ & 0.979 \\
Limiting resolution $(\AA)$ & $47.5-1.9(2.01-1.90)$ \\
Unique reflections & $166608(24184)$ \\
$R_{\text {merge }}(\%)$ & $7.9(43.3)$ \\
$R_{\text {meas }}(\%)$ & $9.3(56.3)$ \\
Multiplicity & $3.3(2.0)$ \\
Completeness $(\%)$ & $96.8(87.1)$ \\
$\langle I / \sigma(I)\rangle$ & $10.82(1.62)$ \\
CC $1 / 2(\%)$ & $99.6(60.1)$ \\
Refinement & \\
Resolution range $(\AA)$ & $30.0-1.90$ \\
Unique reflections, working/free & $158223 / 8261$ \\
$R$ factor $(\%)$ & 18.3 \\
$R_{\text {free }}(\%)$ & 23.76 \\
Non- $\mathrm{H}$ atoms & 14953 \\
Water molecules & 835 \\
R.m.s.d., bonds $(\AA)$ & 0.008 \\
R.m.s.d., angles $\left({ }^{\circ}\right)$ & 1.289 \\
Average $B$ factor $\left(\AA^{2}\right)$ & \\
All atoms & 22.92 \\
Water molecules & 22.49 \\
Zinc ions & 20.20 \\
Bicarbonate ions & 23.81 \\
Ramachandran plot & \\
Preferred $(\%)$ & 94 \\
Allowed $(\%)$ & 6 \\
Outliers $(\%)$ & 0 \\
&
\end{tabular}

identified the $\operatorname{VchCA} \beta$ sequence, with accession No. WP_002051193.1.

\subsection{Construct preparation, protein expression and purification}

The GeneArt company, which specializes in gene synthesis, designed the synthetic $V$. cholerae gene encoding $\beta$-CA and containing four base-pair sequences (CACC) necessary for directional cloning at the $5^{\prime}$ end of the $\operatorname{VchCA} \beta$ gene. The fragment was subsequently cloned into the expression vector pET100/D-TOPO (Invitrogen), creating the plasmid pET-100/ $\mathrm{D}-\mathrm{TOPO} / \mathrm{VchCA} \beta$. In order to confirm the integrity of the $V$. cholerae gene and the fact that no errors occurred at the ligation sites, the vector containing the fragment was sequenced. Competent E. coli BL21 (DE3) cells were transformed with $\mathrm{pET}-100 / \mathrm{D}-\mathrm{TOPO} / \mathrm{V} \operatorname{chCA} \beta$, grown at $37^{\circ} \mathrm{C}$, induced with $1 \mathrm{~m} M$ IPTG and grown for $4 \mathrm{~h}$. After growth for an additional $4 \mathrm{~h}$, the cells were harvested and disrupted by sonication at $4^{\circ} \mathrm{C}$ in $20 \mathrm{~m} M$ phosphate buffer $\mathrm{pH}$ 8.0. Following sonication, the sample was centrifuged at $12000 \mathrm{~g}$ at $4^{\circ} \mathrm{C}$ for $30 \mathrm{~min}$. The supernatant was loaded onto a His-Select HF nickel-affinity column $(1.0 \times 1.0 \mathrm{~cm}$; GE Healthcare $)$ and the column was equilibrated with $0.02 M$ phosphate buffer $\mathrm{pH}$ 8.0 containing $0.02 \mathrm{M}$ imidazole and $0.5 \mathrm{M} \mathrm{NaCl}$ at a flow rate of $1.0 \mathrm{ml} \mathrm{min}{ }^{-1}$. The $\mathrm{VchCA} \beta$ was eluted with $0.02 M$ phosphate buffer $\mathrm{pH} 8.0$ containing $250 \mathrm{mM}$ imidazole and $0.5 \mathrm{M}$ $\mathrm{NaCl}$ at a flow rate of $1.0 \mathrm{ml} \mathrm{min}{ }^{-1}$. Fractions were collected, dialyzed and analyzed by SDS-PAGE (data not shown).
At this stage of purification the enzyme was at least $95 \%$ pure and the yield obtained was $30 \mathrm{mg}$ of recombinant bacterial $\operatorname{VchCA} \beta$.

\subsection{Kinetics methods}

An Applied Photophysics stopped-flow instrument was used to assay the CA-catalyzed $\mathrm{CO}_{2}$ hydration activity (Khalifah, 1971). Bromothymol blue (at a concentration of $0.2 \mathrm{mM}$ ) was used as an indicator, working at an absorbance maximum of $557 \mathrm{~nm}$, with 10-20 $\mathrm{mM}$ Tris $\mathrm{pH} 8.3$ as buffer and $20 \mathrm{mM} \mathrm{Na} \mathrm{SO}_{4}$ to maintain the ionic strength at a constant value (this anion is not inhibitory and has a $K_{\mathrm{i}}$ of $>200 \mathrm{mM}$ against this enzyme), following the initial rates of the CAcatalyzed $\mathrm{CO}_{2}$ hydration reaction for a period of 10-100 s at $25^{\circ} \mathrm{C}$. The $\mathrm{CO}_{2}$ concentration ranged from 1.7 to $17 \mathrm{mM}$ for the determination of the kinetic parameters and inhibition constants. For each measurement, at least six traces of the initial $5-10 \%$ of the reaction were used to determine the initial velocity. Typically, the first $10 \mathrm{~s}$ of the traces were used to determine the initial rate, which was performed by linear fitting using a program provided by Applied Photophysics. The $\mathrm{CO}_{2}$ concentrations ranged from 1.7 to $17 \mathrm{~m} M$ for determination of the kinetic parameters and inhibition constants. The uncatalyzed rates (which needed times of around $30 \mathrm{~s}$ in our assay conditions) were determined in the same manner and were subtracted from the total observed rates. Stock solutions of inhibitor $(1-10 \mathrm{mM})$ were prepared in distilled deionized water and dilutions of up to $0.01 \mu M$ were then made with distilled deionized water. Inhibitor and enzyme solutions were pre-incubated together for $15 \mathrm{~min}$ at room temperature prior to the assay in order to allow the formation of the E-I complex. The inhibition constants were obtained by nonlinear least-squares methods using the Cheng-Prusoff equation, whereas the kinetic parameters for the uninhibited enzymes were obtained from LineweaverBurk plots (Del Prete, De Luca, Vullo et al., 2014; Del Prete et al., 2015; Maresca et al., 2013), as reported previously, and represent the mean of at least three different determinations. The enzyme concentrations at which the measurements were performed were of the order of 9.3-15.6 $\mathrm{nM}$. In these conditions the following Cheng-Prusoff equation was used,

$$
K_{\mathrm{i}}=\frac{\mathrm{IC}_{50}}{1+\frac{[\mathrm{S}]}{K_{\mathrm{m}}}},
$$

where $\mathrm{IC}_{50}$ represents the molarity of inhibitor that reduces the enzyme activity by $50 \%$ (determined using eight different concentrations of inhibitor varying between $0.1 \mathrm{n} M$ and $150 \mu M), K_{\mathrm{m}}$ is the concentration of the substrate at which the enzyme activity is half of the maximum and [S] is the concentration of substrate.

\subsection{Crystallization and data collection}

The enzyme was crystallized at $296 \mathrm{~K}$ using the sitting-drop vapour-diffusion method in 96-well plates (CrystalQuick, Greiner Bio-One, Germany). Drops were prepared using $1 \mu \mathrm{l}$ 
protein solution mixed with $1 \mu$ reservoir solution and were equilibrated against $100 \mu \mathrm{l}$ precipitant solution. The concentration of the protein was $10 \mathrm{mg} \mathrm{ml}^{-1}$ in $50 \mathrm{mM}$ Tris $\mathrm{pH} 7.2$. Initial crystallization condition were found using the JCSGplus screen (Molecular Dimensions) and were optimized. Diffraction-quality crystals grew within one week to approximate dimensions of $0.2 \times 0.2 \times 0.3 \mathrm{~mm}$ from a solution consisting of $8 \%$ PEG 8000, 8\% ethylene glycol, $100 \mathrm{mM}$ HEPES $\mathrm{pH}$ 7.5. The crystals belonged to the primitive tetragonal space group $P 4_{1}$, with unit-cell parameters $a=84.09$, $c=316.38 \AA$. The asymmetric unit contained two tetramers $\left(V_{\mathrm{M}}=2.8 \AA^{3} \mathrm{Da}^{-1}\right.$, solvent content $\left.56.1 \%\right)$ related by pseudotranslational symmetry. A native data set extending to a maximum resolution of $1.9 \AA$ was collected on the BM30A beamline at ESRF, Grenoble, France using a ADSC Quantum 315 r detector and a wavelength of $0.979 \AA$ A. For data collection a crystal of the enzyme was cooled to $100 \mathrm{~K}$ using a solution consisting of $15 \%$ PEG $8000,15 \%$ ethylene glycol, $100 \mathrm{mM}$

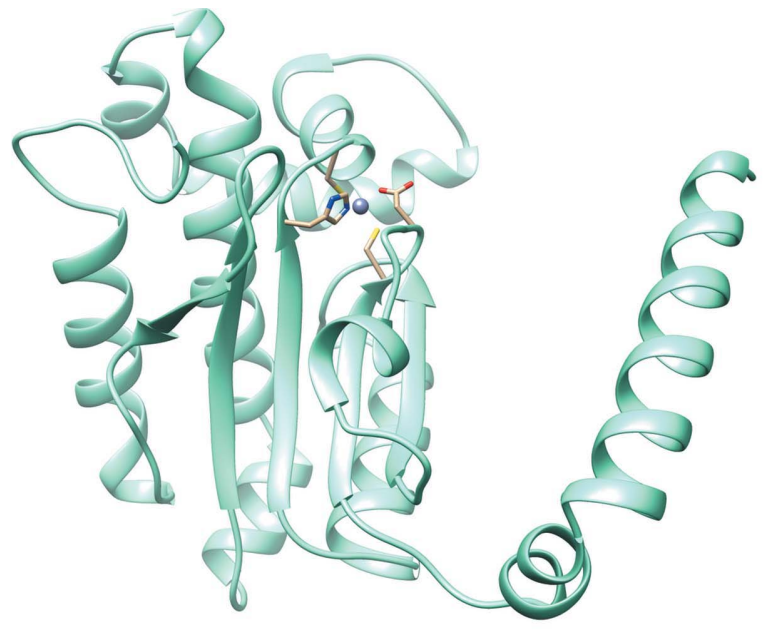

(a)

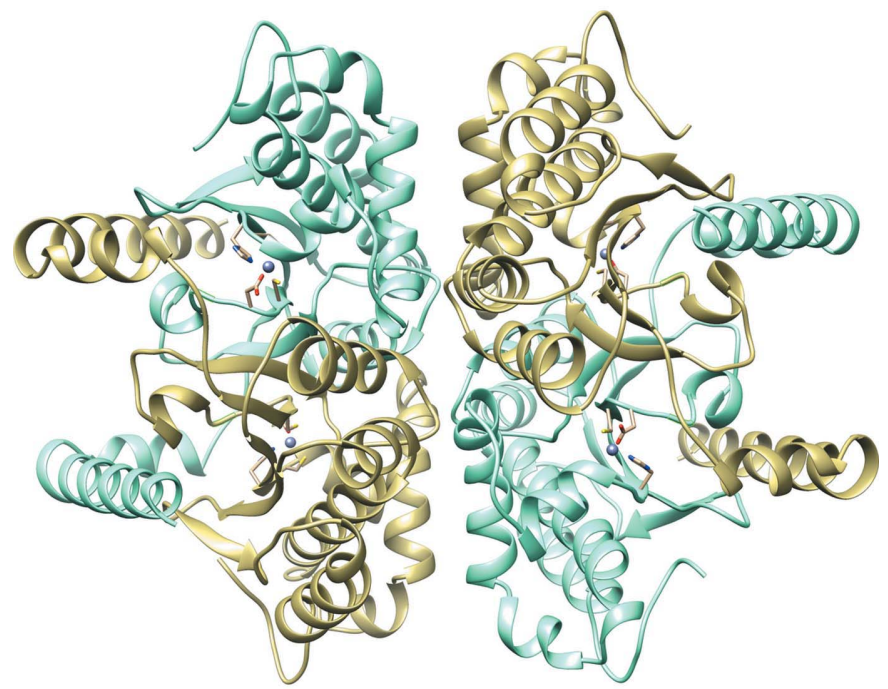

(b)

Figure 1

(a) Ribbon diagram showing a single subunit of the $\operatorname{VchCA} \beta$ structure. The active-site zinc is also represented with its ligands. (b) Ribbon diagram showing the tetrameric arrangement of $\mathrm{VchCA} \beta$.
HEPES pH 7.5 as cryoprotectant. The data were processed with XDS (Kabsch, 2010).

\subsection{Structure determination and refinement}

The structure was solved by the molecular-replacement technique using MOLREP (Vagin \& Teplyakov, 2010) with the coordinates of the structure of $\beta$-carbonic anhydrase from E. coli (PDB entry 2esf; Cronk et al., 2006) as a starting model.

The model was refined using REFMAC5 (Murshudov et al., 2011) from the CCP4 suite (Winn et al., 2011). Manual rebuilding of the model was performed using Coot (Emsley et al., 2010). Solvent molecules were introduced automatically using ARP/wARP (Lamzin et al., 2001). Refinement resulted in $R$-factor and $R_{\text {free }}$ values of 0.183 and 0.238 , respectively. Data processing and refinement statistics are summarized in Table 1. The stereochemistry of the final model was analyzed with PROCHECK (Laskowski et al., 1993). The overall mean $B$ factor of the structure after refinement was $22.92 \AA^{2}$ for water molecules and $22.49 \AA^{2}$ for all atoms. Protein coordinates have been deposited in the Protein Data Bank (PDB entry 5cxk). Structural figures were generated with the UCSF Chimera package (Pettersen et al., 2004).

\subsection{Merohedral twinning}

The data set was initially processed in space group $P 422$ with reasonable statistics $\left(R_{\text {merge }}=8.3 \%\right)$. Molecular replacement in space group $P 4_{1} 22_{1} 2$ provided a solution with a single tetramer in the asymmetric unit, which was refined to an $R$ factor and $R_{\text {free }}$ of 0.23 and 0.27 , respectively. Later it was realised that the data had been collected from a crystal that was almost perfectly merohedrally twinned. Thus, the data were processed again in the lower symmetry tetragonal space group.

A molecular-replacement solution was found in space group $P 4_{1}$ with two tetramers related by a pseudo-translation, which was confirmed by a peak that was $20 \%$ of the origin peak at $(0.0,0.15,0.50)$ in the native Patterson map. No rotational symmetry was detected in the self-rotation function in addition to that derived from the fourfold crystallographic axis and the noncrystallographic 222 symmetry exhibited by one tetramer subunit.

The possibility of twinning of the data was suggested by analysis of the intensity distribution. The phenix.xtriage module of PHENIX (Adams et al., 2002) was used to analyze the data for twinning. The multivariate $Z$-score from the $L$-test of PHENIX was well above the expected value of 3.5. A high $Z$-score value indicates that the data differ significantly from what is expected for untwinned data. The results of the twinning tests and twinning law are shown in Supplementary Table S1.

Thus, the twin-refinement option in REFMAC was used. The $R$ factor and $R_{\text {free }}$ decreased substantially at the stage in which this option was introduced in the refinement. The twinning fraction refined to a value of 0.49 . 


\section{Results and discussion}

\subsection{X-ray structural investigation}

The X-ray structure of VchCA $\beta$ was solved by molecular replacement using the coordinates of the $\beta$-CA from $E$. coli. The enzyme crystallizes with four subunits in the asymmetric unit. Analysis of the crystal contacts using the PISA software (Krissinel \& Henrick, 2007) suggested that the biologically active molecule is a homotetramer possessing 222 symmetry. The final model comprises the entire protein sequence, i.e. residues 1-222, for every chain. Additionally, the model includes eight zinc ions, six bicarbonate ions and 835 water molecules (see Fig. 1).

Among the $\beta$-CAs of known structure, the highest level of sequence homology of $\mathrm{VchCA} \beta$ was found to the $\beta$-CAs from Salmonella enterica (63\% identity), from E. coli (61\% identity) and from $H$. influenzae (58\% identity).

The X-ray structure of $\mathrm{V} \operatorname{chCA} \beta$ is similar to the structures of the other $\beta$-CAs mentioned above. The superposition of the VchCA $\beta$ model with the $\beta$-CAs from $E$. coli (PDB entry 2esf; Cronk et al., 2006), S. enterica (PDB entry 3qy1; Center for Structural Genomics of Infectious Diseases, unpublished work) and $H$. influenzae (PDB entry 2a8d; Cronk et al., 2006) yielded r.m.s.d.s between $\mathrm{C}^{\alpha}$ atoms of $1.202,1.371$ and $1.435 \AA$, respectively (see Fig. 2). Each monomer adopted an $\alpha / \beta$ fold, being composed of an N-terminal arm, which extends away from the rest of the molecule and makes significant contacts with an adjacent monomer, a conserved zinc-binding core and a C-terminal domain dominated by $\alpha$-helices. The zinc-binding core resembles the classical nucleotide-binding Rossmannfold motif (Rossmann et al., 1974) with a $\beta$-sheet composed of four parallel strands flanked on both sides by $\alpha$-helical segments. The active site is located at one end of the central $\beta$-sheet at the interface of one dimer.

In the active site the zinc ion is coordinated to four aminoacid residues, Cys42, Asp44, His98 and Cys101, with an

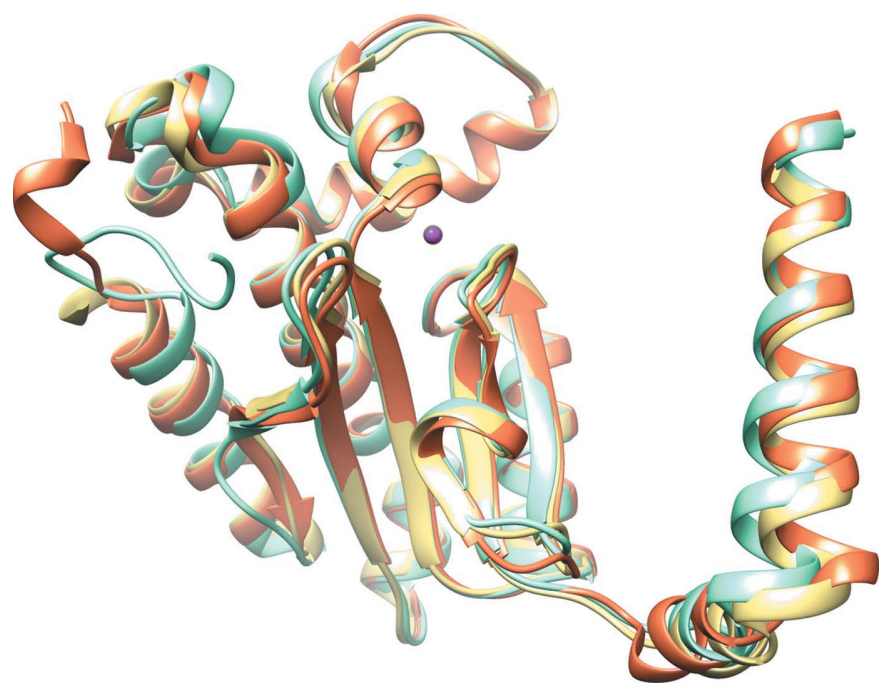

Figure 2

Superposition of a monomer of $\operatorname{VchCA} \beta$ (orange) with that of $\beta$-CA from E. coli (gold) and H. influenzae (light blue). The catalytic zinc ion is represented as a magenta sphere. approximately tetrahedral geometry (Fig. 3). Therefore, $\mathrm{VchCA} \beta$ belongs to the type II subclass $\beta$-CAs, which are characterized by four protein-derived ligands that coordinate the catalytic zinc ion, in contrast to the type I $\beta$-CAs, which have only two cysteine residues and one histidine residue in the zinc-coordination sphere, with a fourth coordination site occupied by a water molecule/hydroxide ion or a substrate analogue/inhibitor (Rowlett, 2010). The zinc-bound Asp44 residue forms a Asp-Arg dyad in type I $\beta$-CAs, whereas in $\mathrm{V} \operatorname{chCA} \beta$ and in all other type II $\beta$-CAs this dyad interaction is broken so that Asp44 can freely coordinate the zinc while Arg46 is rotated away. As shown by the excellent work of Jones and coworkers (Covarrubias et al., 2005, 2006), at pH values of $>8$ the $\mathrm{COOH}$ moiety of Asp44 and the guanidine moiety of Arg46 interact with each other, forming a strong interaction (salt-like or hydrogen bonds), which liberates the fourth coordination position at the $\mathrm{Zn}^{2+}$ ion, which will coordinate an incoming water molecule. This water molecule is further activated by the Asp-Arg dyad (and the $\mathrm{Zn}^{2+}$ ion), providing the nucleophile used by the enzyme in the catalytic cycle (Supuran, 2012; Covarrubias et al., 2005, 2006).

An electron density that is not explainable by protein or solvent atoms was present in the $F_{\mathrm{o}}-F_{\mathrm{c}}$ maps near the active site of six of the eight subunits (chains $A, B, C, D, H$ and $G$ ). This electron density has a triangular and planar shape and was interpreted as a bicarbonate ion. The bicarbonate ion is bound in a pocket at a distance of roughly $8 \AA$ from the zinc and forms hydrogen bonds to the side chains of Trp39, Arg64 and Tyr181 and to the peptide carbonyl of Val47. Each residue donates one or more hydrogen bonds to the bicarbonate; in addition, two water molecules also form multiple hydrogen bonds to bicarbonate $\mathrm{O}$ atoms (see Fig. 4).

Since the bicarbonate cannot be derived either from the purification or the crystallization process, it should be interpreted as an adventitious ligand. Nevertheless, kinetic studies revealed that bicarbonate is an inhibitor as well as a substrate

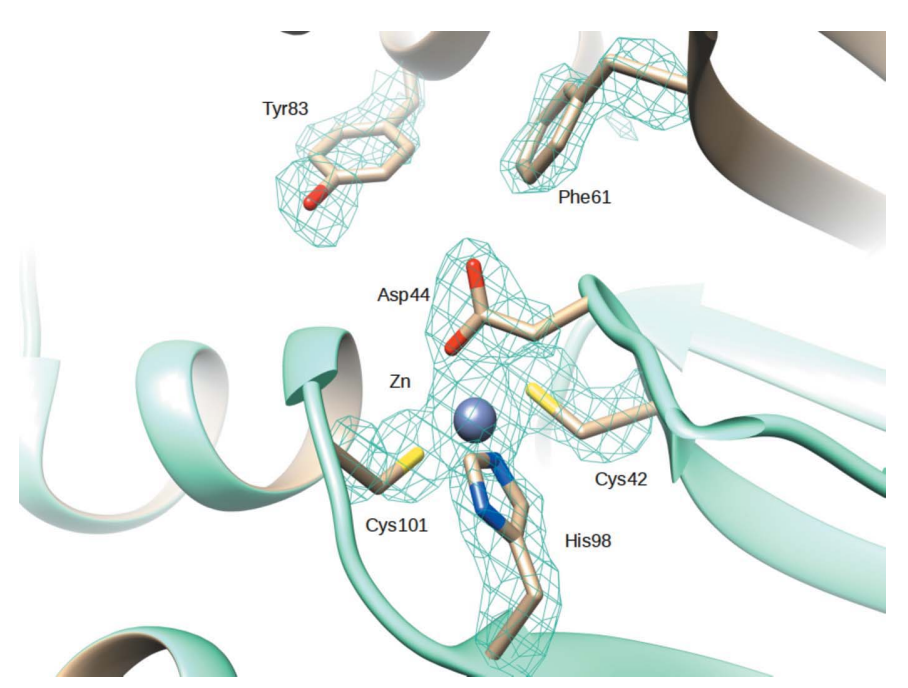

Figure 3

$F_{\mathrm{o}}-F_{\mathrm{c}}$ OMIT map (contoured at $3 \sigma$ ) of the active site of VchCA $\beta$ exhibiting a 'closed' configuration. 
Table 2

Kinetic properties of the $V$. cholerae $\alpha$-class $(\mathrm{VchCA})$ and $\beta$-class $(\mathrm{V} \operatorname{chCA} \beta) \mathrm{CAs}$ for the $\mathrm{CO}_{2}$ hydration reaction determined by a stopped-flow $\mathrm{CO}_{2}$ hydrase assay and their inhibition data with the clinically used sulfonamide acetazolamide (Khalifah, 1971).

Kinetic/inhibition data for other $\alpha$-class and $\beta$-class CAs are given for comparison. The measurements were performed at $\mathrm{pH} 7.5$ for the $\alpha$-CAs and at $\mathrm{pH} 8.3$ for the $\beta$-CAs. Can2, C. neoformans $\beta$-CA; CaNce103, C. albicans $\beta$-CA; psCA3, P. aeruginosa $\beta$-CA, isoform 3 (Supuran, 2012; Pinard et al., 2015).

\begin{tabular}{llllll}
\hline Enzyme & Class & $k_{\text {cat }}\left(\mathrm{s}^{-1}\right)$ & $\begin{array}{l}k_{\text {cat }} / K_{\mathrm{m}} \\
\left(M^{-1} \mathrm{~s}^{-1}\right)\end{array}$ & $\begin{array}{l}K_{\mathrm{i}}(\text { acetazolamide }) \\
(\mathrm{n} M)\end{array}$ & Reference \\
\hline hCA I & $\alpha$ & $2.0 \times 10^{5}$ & $5.0 \times 10^{7}$ & 250 & Supuran (2012) \\
hCA II & $\alpha$ & $1.4 \times 10^{6}$ & $1.5 \times 10^{8}$ & 12 & Supuran (2012) \\
Can2 & $\beta$ & $3.9 \times 10^{5}$ & $4.3 \times 10^{7}$ & 10.5 & Supuran (2012) \\
CaNce103 & $\beta$ & $8.0 \times 10^{5}$ & $9.7 \times 10^{7}$ & 132 & Supuran (2012) \\
PgiCA $\beta$ & $\beta$ & $2.8 \times 10^{5}$ & $1.5 \times 10^{7}$ & 214 & Del Prete, De Luca, Vullo et al. (2014) \\
psCA3 & $\beta$ & $1.40 \times 10^{5}$ & $1.0 \times 10^{7}$ & 75.9 & Pinard et al. (2015) \\
VchCA & $\alpha$ & $8.23 \times 10^{5}$ & $7.0 \times 10^{7}$ & 6.8 & Del Prete, De Luca, Scozzafava et al. (2014) \\
VchCA $\beta$ & $\beta$ & $3.34 \times 10^{5}$ & $4.1 \times 10^{7}$ & 4512 & This work \\
\hline
\end{tabular}

and the concentration of bicarbonate in the crystallization medium, but the kinetic data suggest that these enzymes adopt this conformation in order to function in a highly cooperative manner. The active form can be inferred from the crystal structures of type I $\beta$-CAs in which Asp44 is detached from the catalytic zinc ion and is hydrogenbonded in a bidentate mode to Arg46. Nevertheless, the active form has been observed in two mutants of $H$. influenzae (D44N and P48S/A49P; Rowlett et al., 2009; Hoffmann et al., 2015)

of $H$. influenzae $\beta$-CA, and a bicarbonate ion bound in the same position as in $\mathrm{VchCA} \beta$ has previously been found in two crystal structures: one of $\beta$-CA from $E$. coli (PDB entry 2esf) and another from $H$. influenzae (PDB entry 2a8d) (Cronk et $a l ., 2006)$. It is worth noting that in order to obtain these latter structures the crystals were intentionally soaked in a solution containing $100 \mathrm{mM} \mathrm{NaHCO} 3$ before data collection.

It has already been observed that the binding of the bicarbonate ion to this noncatalytic site enforces a closed conformation of the active site in type II $\beta$-CA structures. In fact bicarbonate expels the side chain of Val47, which causes reorganization of the 44-48 loop and results in the disruption of the Asp44-Arg46 dyad, allowing Asp44 to displace the zincbound water and bind directly to the zinc ion. This state has been called the $\mathrm{T}$ state or inactive state, and it was previously found to be present at acidic and neutral $\mathrm{pH}$. However, the alternative open configuration has not been observed in any type II $\beta$-CA structure, regardless of the crystallization $\mathrm{pH}$

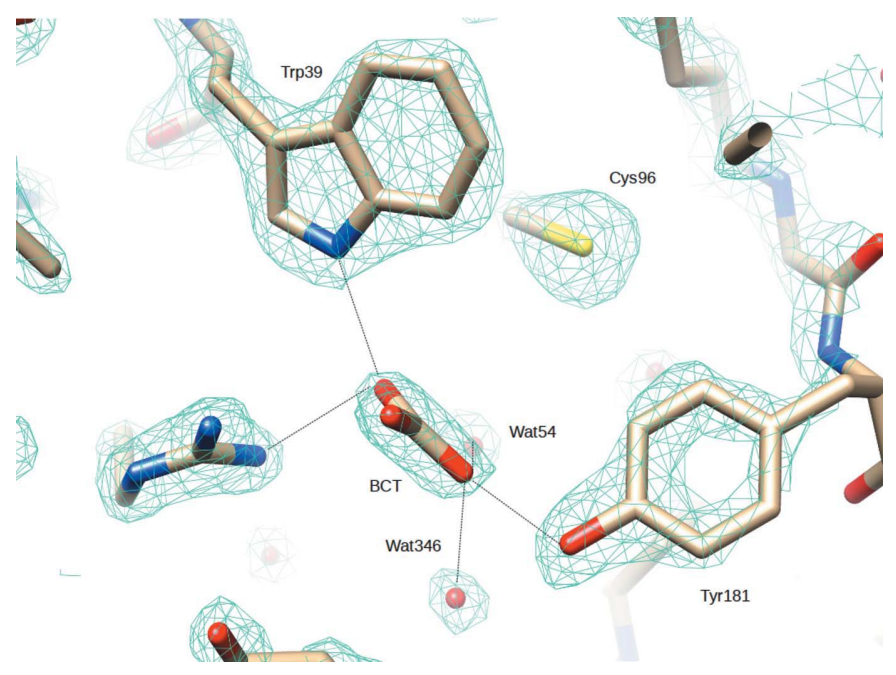

Figure 4

The noncatalytic bicarbonate-binding site. A $2 F_{\mathrm{o}}-F_{\mathrm{c}}$ electron-density map (contoured at $1.5 \sigma$ ) is also shown. Hydrogen bonds formed by the bicarbonate ions to enzyme residues and water molecules are also depicted as dashed lines. and in a thiocyanate inhibitor complex of $M$. tuberculosis $\beta$-CA (Covarrubias et al., 2006).

The presence of a triad of residues formed by Trp39, Tyr181 and Arg64 has previously been recognized as fundamental for the binding of bicarbonate to the noncatalytic site and is strictly conserved in type II $\beta$-CAs. In contrast, the Trp-ArgTyr triad is absent in all type I $\beta$-CAs. Furthermore, the noncatalytic bicarbonate-binding site seems to be especially evolved for this particular ion, as other isoelectronic ions such as acetate or nitrate would not be able to achieve all of the hydrogen-bonding interactions in which bicarbonate participates simultaneously. Thus, our findings reinforce the hypothesis that the bicarbonate binding to a noncatalytic site near the zinc ion is a distinctive feature of type II $\beta$-CAs and plays a significant role in the functionality of this enzyme type.

\subsection{Kinetic data}

We have investigated the kinetic properties of $\operatorname{VchCA} \beta$ as a catalyst for the hydration of carbon dioxide to bicarbonate at $\mathrm{pH} 8.3$, when presumably the active site of the enzyme should be in the open state, i.e. with water or hydroxide as the fourth zinc ligand. Data are reported in Table 2 , in which we have included, for comparison, kinetic data for other $\alpha$ - and $\beta$-class CAs of various origins, such as the bacterial CA from the same pathogen belonging to the $\alpha$-class (VchCA; Del Prete, De Luca, Scozzafava et al., 2014), but also the human main red blood cell isoforms hCA I and II (Supuran, 2012; Khalifah, 1971), together with $\beta$-CAs from various pathogens, such as Can2 from the fungus Cryptococcus neoformans, CaNce103 from Candida albicans, psCA3 from the bacterium Pseudomonas aeruginosa (Supuran, 2012; Pinard et al., 2015) and $\operatorname{PgiCA} \beta$ from another bacterial pathogen, Porphyromonas gingivalis (Del Prete, De Luca, Vullo et al., 2014). The $\mathrm{CO}_{2}$ hydration reaction was monitored with and without a catalyst by a stopped-flow $\mathrm{CO}_{2}$ hydration assay at $\mathrm{pH} 7.5$ for the $\alpha$-CAs and at $\mathrm{pH} 8.3$ for the $\beta$-CAs. Inhibition data with the clinically used sulfonamide acetazolamide are also given (Khalifah, 1971). 
The data in Table 2 show that $\mathrm{VchCA} \beta$ possesses a good catalytic activity for the physiological reaction, with kinetic parameters similar to those of the slow human isoform hCA I or the pathogenic enzymes from $C$. neoformans ( $\beta$-class) or $V$. cholerae $(\alpha-\mathrm{CA})$. Indeed, with a $k_{\text {cat }}$ of $3.34 \times 10^{5} \mathrm{~s}^{-1}$ and a $k_{\text {cat }} / K_{\mathrm{m}}$ of $4.1 \times 10^{7} \mathrm{M}^{-1} \mathrm{~s}^{-1}$, this enzyme is much more efficient as a catalyst compared with psCA3 and $\operatorname{PgiCA} \beta$, only being less effective when compared with hCA II (one of the best catalysts known in nature) and CaNce103 among the enzymes shown in Table 2 (Del Prete, De Luca, Vullo et al., 2014; Del Prete et al., 2015; Maresca et al., 2013). However, unlike most of the enzymes discussed above, $\mathrm{V} \operatorname{chCA} \beta$ was only poorly inhibited by the classical sulfonamide CA inhibitor used clinically, acetazolamide, with an inhibition constant of $>4.5 \mu M$ (Table 2). It should be noted that many of the CAs discussed here are much more effectively inhibited by this sulfonamide, with $K_{\mathrm{i}}$ values ranging between 6.8 and $250 \mathrm{n} M$.

This good catalytic activity of $\mathrm{VchCA} \beta$ can of course be easily explained considering the crystallographic data presented above as well as the alignment of various bacterial $\beta$-CAs shown in Fig. 5. Indeed, $\mathrm{V} \operatorname{chCA} \beta$ possesses all of the conserved aminoacid residues essential for catalysis, such as, for example, the zinc(II) ligands (Cys42, His98 and Cys101 in the open active-site state) as well as the catalytic dyad (Asp44-Arg46) present in all of these enzymes irrespective whether they belong to the type I or type II subclass.

\section{Conclusions}

The cloning, X-ray crystal data and kinetic characterization of the $\beta$-CA from $V$. cholerae $(\mathrm{VchCA} \beta)$ are reported in this paper. The X-ray crystal structure of the enzyme was solved at $1.9 \AA$ resolution. $\mathrm{V}$ chCA $\beta$ was revealed to be a tetrameric type II $\beta$-CA with a closed active site in which the zinc is tetrahedrally coordinated to Cys42, Asp44, His98 and Cys101 (the crystallization was performed at a $\mathrm{pH}$ of 7.5). Furthermore, the substrate bicarbonate was observed bound in each monomer of $\mathrm{VchCA} \beta$ in a noncatalytic binding pocket close to the zinc ion, as reported for a few other $\beta$-CAs, such as those from $E$. coli and $H$. influenzae. At $\mathrm{pH} 8.3$ the enzyme showed a significant catalytic activity for the physiological reaction of the hydration of $\mathrm{CO}_{2}$ to bicarbonate and protons, with the

Figure 5

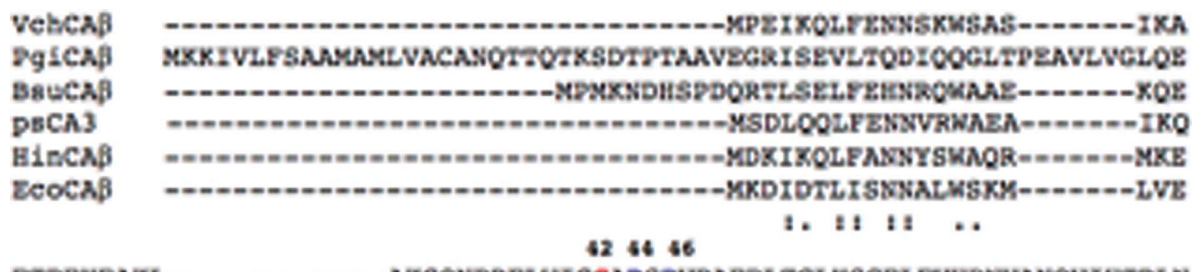

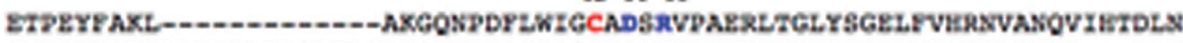
GNARYVAXKQLPRDLNAQAVAGLEGQFEAI ILSCIDSRVPVEYIYDKGIGDLFVGRVAGNVV---DDE

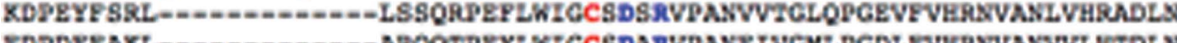

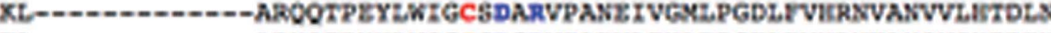

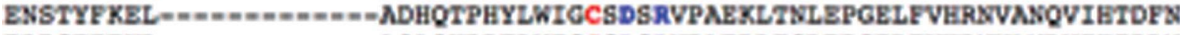

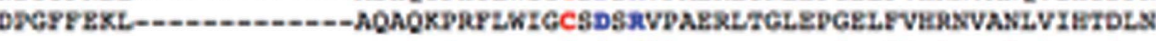

CLSVVQYAVDVLQVKHI IVCGHYCCCOVTMIDNPQLCLINYWLLHIRDYYLKHREYLDKMPA-EDRSD

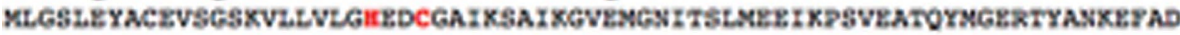
LLSVLEFAVGVLEIKHI IVCGBYGCGCVRAAMDCYGHGI IDNWLQP IRDIAQANQAELDZIENTQDRLD CLSVIQYAVDVLKVKHILVTGIYCCCCVIASLRNDOLCLYDGWLRSYRDLAYZYYEHLEQLPTEEESVD

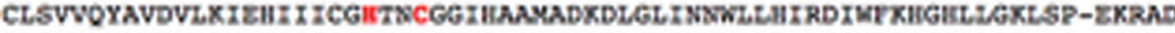
CSWVQYAVDVLEVEHII ICGHYCCCOVQNAVENPELCLINVWLLHIRD IMFKHSSLLDEMPQ-BRRLD

KLAEINVAEQVYNLANSTVLONANESCQAVEVEGFVYCYEDGRLYLGVRCASRSAVEDWYHKALE--X AVVKZNVICFXDEIRRDSPILXKLEZEGXIXICGAIYEMSTGKVIYZRLCELSVSSQVESLSREPVLQSANKDCKDI IVECWYYNLKDGLLRDIGCDCT-RNALQFACQRNE- - -

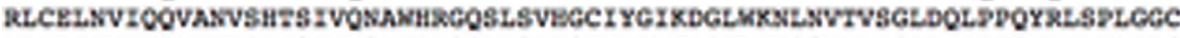
YLTKTNVAEQVYNLGRTSTVKSANERGOKLSLEGWVYDVNDGFLVDOGVMATSRETLEISYRNATARLS

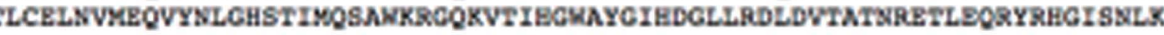

ILspsHaLLCR-----

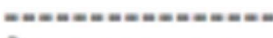

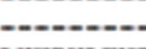

LKEANHK-

Alignment of the amino-acid sequences of $\beta$-CAs from different bacteria. Zinc ligands are indicated substitutions are observed. Multi-alignment was performed with Muscle. Legend: VchCA $\beta$, Vibrio cholerae (accession No. WP_002051193.1); $\operatorname{PgiCA} \beta$, Porphyromonas gingivalis (accession No. WP_021663681.1); BsuCA $\beta$, Brucella suis (accession No. AAN33967.1); psCA3, Pseudomonas aeruginosa PA01 (accession No. GI:848130166); HinCA $\beta$, Haemophilus influenzae (accession No. WP_044345628.1); EcoCA $\beta$, Escherichia coli (accession No. WP_000651595.1).

following kinetic parameters: a $k_{\text {cat }}$ of $3.34 \times 10^{5} \mathrm{~s}^{-1}$ and a $k_{\text {cat }} /$ $K_{\mathrm{m}}$ of $4.1 \times 10^{7} \mathrm{M}^{-1} \mathrm{~s}^{-1}$. The new enzyme was, on the other hand, poorly inhibited by acetazolamide $\left(K_{\mathrm{i}}\right.$ of $\left.4.5 \mu M\right)$. As this bacterial pathogen encodes at least three CAs, an $\alpha$-CA, a $\beta$-CA and a $\gamma$-CA, these enzymes probably play important roles in the life cycle and pathogenicity of Vibrio, and it cannot be excluded that interference with their activity may be exploited therapeutically to obtain antibiotics with a different mechanism of action.

\section{Acknowledgements}

The research leading to these results received funding from the European Community's Seventh Framework Programme (FP7/2007-2013) under BioStruct-X (grant agreement No. 283570).

\section{References}

Abuaita, B. H. \& Withey, J. H. (2009). Infect. Immun. 77, 4111-4120. 
Adams, P. D., Grosse-Kunstleve, R. W., Hung, L.-W., Ioerger, T. R., McCoy, A. J., Moriarty, N. W., Read, R. J., Sacchettini, J. C., Sauter, N. K. \& Terwilliger, T. C. (2002). Acta Cryst. D58, 1948-1954.

Aggarwal, M., Boone, C. D., Kondeti, B. \& McKenna, R. (2013). J. Enzyme Inhib. Med. Chem. 28, 267-277.

Aggarwal, M., Kondeti, B. \& McKenna, R. J. (2013). Bioorg. Med. Chem. 21, 1526-1533.

Capasso, C. \& Supuran, C. T. (2013). Expert Opin. Ther. Pat. 23, 693-704.

Capasso, C. \& Supuran, C. T. (2015). J. Enzyme Inhib. Med. Chem. 30, 325-332.

Covarrubias, A. S., Bergfors, T., Jones, T. A. \& Hogbom, M. (2006). J. Biol. Chem. 281, 4993-4999.

Covarrubias, A. S., Larsson, A. M., Högbom, M., Lindberg, J., Bergfors, T., Björkelid, C., Mowbray, S. L., Unge, T. \& Jones, T. A. (2005). J. Biol. Chem. 280, 18782-18789.

Cronk, J. D., Endrizzi, J. A., Cronk, M. R. W., O'Neill, J. \& Zhang, K. Y. J. (2001). Protein Sci. 10, 911-922.

Cronk, J. D., Rowlett, R. S., Zhang, K. Y. J., Tu, C., Endrizzi, J. A., Lee, J., Gareiss, C. \& Preiss, J. R. (2006). Biochemistry, 45, 4351-4361.

Del Prete, S., De Luca, V., Scozzafava, A., Carginale, V., Supuran, C. T. \& Capasso, C. (2014). J. Enzyme Inhib. Med. Chem. 29, 23-27.

Del Prete, S., De Luca, V., Vullo, D., Scozzafava, A., Carginale, V., Supuran, C. T. \& Capasso, C. (2014). J. Enzyme Inhib. Med. Chem. 29, 532-537.

Del Prete, S., Vullo, D., De Luca, V., AlOthman, Z., Osman, S. M., Supuran, C. T. \& Capasso, C. (2015). J. Enzyme Inhib. Med. Chem. 30, 366-370.

Emsley, P., Lohkamp, B., Scott, W. G. \& Cowtan, K. (2010). Acta Cryst. D66, 486-501.

Harris, J. B., LaRocque, R. C., Qadri, F., Ryan, E. T. \& Calderwood, S. B. (2012). Lancet, 379, 2466-2476.

Heidelberg, J. F. et al. (2000). Nature (London), 406, 477-483.

Hoffmann, K. M., Million-Perez, H. R., Merkhofer, R., Nicholson, H. \& Rowlett, R. S. (2015). Biochemistry, 54, 598-611.

Huang, S., Hainzl, T., Grundström, C., Forsman, C., Samuelsson, G. \& Sauer-Eriksson, A. E. (2011). PloS One 6, e28458.

Kabsch, W. (2010). Acta Cryst. D66, 133-144.

Khalifah, R. G. (1971). J. Biol. Chem. 246, 2561-2573.

Kimber, M. S. \& Pai, E. F. (2000). EMBO J. 19, 1407-1418.
Krissinel, E. \& Henrick, K. (2007). J. Mol. Biol. 372, 774-797.

Lamzin, V. S., Perrakis, A. \& Wilson, K. S. (2001). International Tables for Crystallography, Vol. F, edited by M. G. Rossmann \& E. Arnold, pp. 720-722. Dordrecht: Kluwer Academic Publishers.

Laskowski, R. A., MacArthur, M. W., Moss, D. S. \& Thornton, J. M. (1993). J. Appl. Cryst. 26, 283-291.

Lehneck, R., Neumann, P., Vullo, D., Elleuche, S., Supuran, C. T., Ficner, R. \& Pöggeler, S. (2014). FEBS J. 281, 1759-1772.

Lehneck, R. \& Pöggeler, S. (2014). Appl. Microbiol. Biotechnol. 98, 8433-8441.

Maresca, A., Carta, F., Vullo, D. \& Supuran, C. T. (2013). J. Enzyme Inhib. Med. Chem. 28, 407-411.

Mitsuhashi, S., Mizushima, T., Yamashita, E., Yamamoto, M., Kumasaka, T., Moriyama, H., Ueki, T., Miyachi, S. \& Tsukihara, T. (2000). J. Biol. Chem. 275, 5521-5526.

Murshudov, G. N., Skubák, P., Lebedev, A. A., Pannu, N. S., Steiner, R. A., Nicholls, R. A., Winn, M. D., Long, F. \& Vagin, A. A. (2011). Acta Cryst. D67, 355-367.

Pettersen, E. F., Goddard, T. D., Huang, C. C., Couch, G. S., Greenblatt, D. M., Meng, E. C. \& Ferrin, T. E. (2004). J. Comput. Chem. 25, 1605-1612.

Pinard, M. A., Lotlikar, S. R., Boone, C. D., Vullo, D., Supuran, C. T., Patrauchan, M. A. \& McKenna, R. (2015). Bioorg. Med. Chem. 23, 4831-4838.

Rossmann, M. G., Moras, D. \& Olsen, K. W. (1974). Nature (London), 250, 194-199.

Rowlett, R. S. (2010). Biochim. Biophys. Acta, 1804, 362-373.

Rowlett, R. S., Tu, C., Lee, J., Herman, A. G., Chapnick, D. A., Shah, S. H. \& Gareiss, P. C. (2009). Biochemistry, 48, 6146-6156.

Rowlett, R. S., Tu, C., McKay, M. M., Preiss, J. R., Loomis, R. J., Hicks, K. A., Marchione, R. J., Strong, J. A., Donovan, G. S. Jr \& Chamberlin, J. E. (2002). Arch. Biochem. Biophys. 404, 197209.

Sawaya, M. R., Cannon, G. C., Heinhorst, S., Tanaka, S., Williams, E. B., Yeates, T. O. \& Kerfeld, C. A. (2006). J. Biol. Chem. 281, 7546-7555.

Strop, P., Smith, K. S., Iverson, T. M., Ferry, J. G. \& Rees, D. C. (2001). J. Biol. Chem. 276, 10299-10305.

Supuran, C. T. (2012). J. Enzyme Inhib. Med. Chem. 27, 759-772.

Vagin, A. \& Teplyakov, A. (2010). Acta Cryst. D66, 22-25.

Winn, M. D. et al. (2011). Acta Cryst. D67, 235-242. 